\title{
Creencias relacionadas al cuidado de la salud oral en cuidadores de adultos mayores institucionalizados de la ciudad de Antofagasta, Chile, 2019
}

\author{
Oral health care related beliefs among caregivers of \\ institutionalized elderly in Antofagasta city, Chile, 2019
}

Crenças relacionadas à atenção à saúde bucal em cuidadores de idosos institucionalizados na cidade de Antofagasta, Chile, 2019

\author{
Juan Godoy' (D) 0000-0003-3057-6281 \\ Elizabeth Rosales ${ }^{2}$ (DD 0000-0003-3542-7074 \\ Constanza Garrido-Urrutia² (iD) 0000-0002-4341-5762
}

\section{Resumen}

Objetivo: analizar las creencias relacionadas al cuidado de la salud oral en cuidadores de adultos mayores institucionalizados de la ciudad de Antofagasta, Chile, 2019.

Material y Métodos: Estudio cuantitativo, observacional, descriptivo, transversal. Se entrevistó a 49 cuidadores con el cuestionario DCBS-sp, para determinar sus creencias relacionadas al cuidado de la salud oral. Para análisis estadístico se utilizó STATA $14^{\circ}$.

Resultados: El 36,73\% tiene capacitación en salud oral y el $97,96 \%$, percibe la necesidad de capacitarse. Cuidadores con capacitación tienen significativamente más puntajes promedio favorables a la salud bucal en las dimensiones locus control interno y autoeficacia.

Conclusiones: Cuidadores tienen baja capacitación para el cuidado de la salud bucal, existiendo creencias desfavorables al cuidado de la salud bucal de las personas mayores institucionalizados. Es necesario diseñar intervenciones de educación para la salud bucal en este grupo que permitan mejorar las creencias relacionadas al cuidado de la salud oral.

Palabras clave: creencias en salud oral, cuidadores, cuidado bucal.

Escuela de Odontología, Universidad Pedro de Valdivia, Chile

Departamento de Odontología, Universidad de Antofagasta, Chile 


\section{Abstract}

Objective: to analyze oral health care related beliefs among institutionalized elderly caregivers in Antofagasta city, Chile, 2019.

Material and Methods: A quantitative, observational, descriptive, cross-sectional study was conducted. 49 caregivers were interviewed with the DCBS-sp questionnaire to determine their oral health care related beliefs. For statistical analysis, STATA $14^{\circ}$ was used.

Results: $36.73 \%$ have training in oral health and $97.96 \%$ perceive the need for training. Trained caregivers have significantly more favorable oral health scores on the internal locus control and self-efficacy dimensions.

Conclusions: There is low training for oral health care in this group, there are unfavorable beliefs about oral health care for institutionalized older adults. It is necessary to design oral health educational interventions in this group to improve oral health care related beliefs.

Keywords: oral health beliefs, caregivers, oral care.

\section{Introducción y antecedentes}

De acuerdo con la literatura, los adultos mayores institucionalizados corresponden a personas mayores en estado de fragilidad o dependencia, que ven reducida su capacidad para mantener su salud bucal, debido a la presencia de deterioro cognitivo, pérdida de funcionalidad y/o autonomía ${ }^{(1)}$, por lo cual requieren apoyo o ayuda de otra persona para realizar actividades básicas de la vida cotidiana, como por ejemplo el cepillado de dientes, en dónde el cuidador es

\section{Resumo}

Objetivo: analisar as crenças relacionadas à atenção à saúde bucal em cuidadores de idosos institucionalizados na cidade de Antofagasta, Chile, 2019.

Materiais e Métodos: Estudo quantitativo, observacional, descritivo, transversal. 49 cuidadores foram entrevistados com o questionário DCBS-sp para determinar suas crenças relacionadas aos cuidados com a saúde bucal. Para análise estatística, foi utilizado o STATA 14 .

Resultados: $36,73 \%$ possuem treinamento em saúde bucal e 97,96\% percebem a necessidade de treinamento. Cuidadores treinados apresentam escores médios significativamente mais elevados favoráveis à saúde bucal nas dimensóes de controle do locus interno e autoeficácia.

Conclusóes: Os cuidadores apresentam baixa formação para os cuidados com a saúde bucal, há crenças desfavoráveis em relação aos cuidados com a saúde bucal de idosos institucionalizados. É necessário desenhar intervençôes de educação em saúde bucal nesse grupo para melhorar as crenças relacionadas aos cuidados com a saúde bucal.

Palavras-chave: crenças em saúde bucal, cuidadores, cuidados bucais.

responsable parcial o totalmente del desarrollo de esta práctica ${ }^{(2)}$. Lo anterior impacta en la salud oral de las personas institucionalizadas, existiendo evidencia de que el deterioro del estado de salud bucal es significativamente mayor en personas mayores institucionalizadas y dependientes, comparado con sus contrapartes que mantienen la autonomía ${ }^{(3)}$.

Aunque el rol del cuidador formal en el cuidado bucal del adulto mayor institucionalizado es trascendental en la mantención de su salud bucal, se han reportado barreras para la reali- 
zación de los procedimientos de higiene bucal, por ejemplo, que el adulto mayor no quiere que otra persona realice la higiene de su boca o no sigue las instrucciones que le entrega el cuidador, entre otras ${ }^{(4)}$. Por otro lado, también se ha descrito que parte de las barreras a las que se enfrenta el cuidador al realizar labores de higiene bucal de un tercero dependen del valor que el cuidador otorga a su propia salud bucal y a la salud bucal de las personas que están bajo su cuidado ${ }^{(5)}$.

Lo anterior, muestra la necesidad de implementar programas educativos en salud bucal enfocados en los cuidadores, abordando las barreras existentes para la realización del cuidado bucal. De hecho, se ha demostrado que este tipo de intervenciones en los cuidadores son efectivas, ya que mejoran los indicadores de higiene bucal de las personas mayores que están bajo su cargo ${ }^{(1,2)}$. En este aspecto, se ha impulsado que en el diseño de programas educativos en salud bucal se considere un diagnóstico previo de la comunidad o grupo a intervenir con la finalidad de poder identificar necesidades, comprender la experiencia y la vivencia de quienes serán beneficiarios del programa ${ }^{(6)}$.

La evidencia en Chile respecto a esta problemática es escasa y proviene de localidades en el centro y sur del país, sin encontrarse estudios al respecto en el norte del país. Sin embargo, los estudios publicados son consistentes con lo encontrado a nivel internacional, reportándose una alta prevalencia de enfermedades bucales en personas mayores dependientes domiciliarias, mal estado de sus prótesis dentales y mala higiene bucal salud bucal ${ }^{(7,8,9)}$. Un estudio en cuidadores dependientes domiciliarios demostró que a medida que el nivel de dependencia del adulto mayor aumenta, el rol del cuidador en la higiene bucal del paciente domiciliario adquiere más importancia, ya que la persona mayor es incapaz de realizarla por sí misma. En este estudio también se identificó baja frecuencia de capacitación para el cuidado bucal, en dónde el 73,3\% de ellos no ha recibido capaci- tación ${ }^{(4)}$. Esto también es una realidad que se ha observado en cuidadores de adultos mayores institucionalizados que dónde más del $70 \%$ de ellos no han recibido capacitación para realizar la higiene bucal diaria ${ }^{(10,11)}$ y en dónde se reporta que el $92 \%$ de las personas mayores institucionalizadas necesitan ayuda en la higiene bucal (11). Es por esto por lo que, el desafío de este proyecto de investigación fue analizar las creencias relacionadas al cuidado de la salud oral de los cuidadores de adultos mayores institucionalizados de la ciudad de Antofagasta, en el norte de Chile. Esta evidencia será útil, para el diseño y posterior implementación de intervenciones de apoyo y capacitación de estos cuidadores.

\section{Materiales y Métodos}

Contándose con la certificación del Comité de Ética de la Universidad Pedro de Valdivia y la autorización de los directores técnicos de los establecimientos de larga estadía (ELEAM) de la ciudad de Antofagasta en la segunda región de Chile. Se realizó un estudio cuantitativo, observacional, descriptivo, transversal. La población objetivo correspondió a los cuidadores de adultos mayores que estuvieran trabajando en los ELEAM de la ciudad de Antofagasta en el año 2019. Se consideró a la totalidad de la población de cuidadores, excluyéndose a quienes se negaron a participar en el estudio o llevaban trabajando como cuidadores menos de dos meses. Del total de cuidadores, la totalidad de ellos aceptó participar y solo se excluyó uno por llevar trabajando dos días al momento de la medición, resultando en una muestra de 49 sujetos, que accedieron a participar y firmaron consentimiento informado.

Se llevó a cabo una entrevista realizada por un único investigador, recabando información respecto a las características de los cuidadores en términos de edad, sexo, nivel educacional, cuánto tiempo lleva siendo cuidador de adultos mayores, si tenían o no capacitación en salud oral y su autopercepción de necesidad de capacitación 
para realizar el cuidado bucal de una persona mayor. Además, se realizaron las preguntas del cuestionario DCBS-sp, traducido y validado en Chile por Garrido-Urrutia C et al. ${ }^{(12)}$ que busca determinar las creencias relacionadas a la salud bucal de personas que están a cargo del cuidado bucal de terceros. Este cuestionario consta de 28 preguntas ordenadas al azar y 4 dimensiones, cada una constituida por 7 preguntas. Las respuestas del cuestionario van en una escala de 5 puntos, dónde 1 denota creencias positivas y 5 creencias negativas ${ }^{(12)}$. La multidimensionalidad de este instrumento permite distinguir diferentes variables sicológicas que influyen en las conductas en salud. La dimensión locus de control interno, dice relación a las creencias de que un resultado depende de las propias capacidades, conductas o responsabilidades; la dimensión locus de control externo, se refiere a creencias de que un resultado depende de factores externos que el individuo no puede controlar; la dimensión autoeficacia, corresponde a la autopercepción de capacidad del individuo y finalmente la dimensión de creencias en salud oral se refiere a creencias en aspectos de la salud oral ${ }^{(13)}$.
Los datos recolectados fueron analizados con el software estadístico STATA $14^{\circledR}$. Se realizó un análisis descriptivo de las variables estudiadas, resumiéndolas mediante porcentaje, promedios y desviación estándar (DS). Para el análisis de diferencia de medias por dimensión entre cuidadores que reportaban tener capacitación en salud oral y quienes no la tenían, se utilizó el test T-Student con un $95 \%$ de confianza y un $\mathrm{p}<0,05$.

\section{Resultados}

En la Tabla I, se observan las características sociodemográficas de edad, nivel educacional, tiempo como cuidador, capacitación en salud oral y autopercepción de necesidad de capacitación para realizar el cuidado bucal de un adulto mayor. La totalidad de los cuidadores formales que participaron en la investigación correspondían a mujeres. La mayoría de los cuidadores perciben la necesidad de recibir capacitación para realizar el cuidado bucal de un adulto mayor, ya que solo uno de ellos dijo que no percibía necesitarla. Solo 18 cuidadores (36,73\%) han recibido capacitación en salud oral.

Tabla 1: Características sociodemográficas de cuidadores de los ELEAM de Antofagasta, Chile, 2019 ( $n=49)$.

\begin{tabular}{|l|l|c|c|}
\hline Variables & & $\mathbf{n}$ & $\mathbf{( \% )}$ \\
\hline Edad & Promedio 39,02 (DS 13,2) & & \\
\hline Nivel educacional & Básica incompleta & 5 & $(10,2)$ \\
\hline & Básica completa & 2 & $(4,08)$ \\
\hline & Media incompleta & 8 & $(16,33)$ \\
\hline & Media completa & 18 & $(36,73)$ \\
\hline & Superior incompleta & 5 & $(10,2)$ \\
\hline & Superior completa & 11 & $(22,45)$ \\
\hline Tiempo como cuidador (años) & Promedio 3,69 (DS 4,61) & & \\
\hline & Menos de 1 año & 8 & $(16,33)$ \\
\hline & Entre 1 y 2 años & 23 & $(46,94)$ \\
\hline Capacitación en salud oral & Más de 3 años & 18 & $(36,73)$ \\
\hline Autopercepción de necesidad de capacitación para & Si & 18 & $(36,73)$ \\
\hline realizar el cuidado bucal de una persona mayor & No & 31 & $(63,27)$ \\
\hline & Si & 48 & $(97,96)$ \\
\hline
\end{tabular}


En la Tabla II, se observan los porcentajes de respuesta por pregunta y puntaje promedio por pregunta del cuestionario de creencias relacionadas al cuidado de la salud oral en los cuidadores (DCBS-sp).

Finalmente, la Tabla III, muestra el puntaje promedio por dimensión del cuestionario DCBS- sp y según si el cuidador tenía o no capacitación en salud oral. Los cuidadores que han recibido capacitación tienen significativamente promedios inferiores, es decir, favorables a la salud bu$\mathrm{cal}$, en las dimensiones locus control interno y autoeficacia $(\mathrm{p}<0,05)$.

Tabla 2: Porcentaje de respuestas y puntaje promedio por pregunta del cuestionario DCBS-sp (n=49)

\begin{tabular}{|c|c|c|c|c|c|c|c|}
\hline Preguntas & $\begin{array}{l}\text { Dimen- } \\
\text { sión }\end{array}$ & $\begin{array}{c}\text { Totalmente } \\
\text { de acuerdo } \\
(\%)\end{array}$ & $\begin{array}{l}\text { De } \\
\text { acuerdo } \\
(\%)\end{array}$ & $\begin{array}{l}\text { No } \\
\text { sabe } \\
\text { (\%) }\end{array}$ & $\begin{array}{l}\text { Desacuer- } \\
\text { do (\%) }\end{array}$ & $\begin{array}{l}\text { Totalmente } \\
\text { desacuerdo } \\
\text { (\%) }\end{array}$ & $\begin{array}{l}\text { Puntaje } \\
\text { promedio } \\
\text { (DS) }\end{array}$ \\
\hline $\begin{array}{l}\text { 1. Creo que son los mismos pacientes los } \\
\text { que avisan cuando necesitan ayuda en el } \\
\text { cuidado de su salud bucal }\end{array}$ & CSO & 40,82 & 14,29 & 6,12 & 26,53 & 12,24 & $3,44(1,54)$ \\
\hline $\begin{array}{l}\text { 2. Si les cepillo los dientes y uso la seda den- } \\
\text { tal correctamente, espero menos problemas } \\
\text { dentales }\end{array}$ & AE & 55,1 & 24,49 & 10,2 & 8,16 & 2,04 & $1,77(1,06)$ \\
\hline $\begin{array}{l}\text { 3. Creo que sé cómo se pueden tratar las } \\
\text { diferentes enfermedades de la mucosa bucal }\end{array}$ & $\mathrm{AE}$ & 4,08 & 8,16 & 71,43 & 14,29 & 2,04 & $3,02(0,69)$ \\
\hline $\begin{array}{l}\text { 4. Creo que los productos fluorados son más } \\
\text { apropiados para los niños }\end{array}$ & CSO & 20,41 & 20,41 & 36,73 & 14,29 & 8,16 & $3,3(1,19)$ \\
\hline $\begin{array}{l}\text { 5.Creo que los dientes deberían durar toda } \\
\text { la vida }\end{array}$ & $\mathrm{LCl}$ & 48,98 & 30,61 & 4,08 & 8,16 & 8,16 & $1,95(1,27)$ \\
\hline $\begin{array}{l}\text { 6. Sólo los dentistas pueden prevenir las } \\
\text { caries y las enfermedades de las encías }\end{array}$ & LCE & 14,29 & 18,37 & 4,08 & 48,98 & 14,29 & $2,69(1,32)$ \\
\hline 7. Creo que las caries pueden prevenirse & $\mathrm{LCl}$ & 71,43 & 22,45 & 6,12 & 0 & 0 & $1,34(0,59)$ \\
\hline $\begin{array}{l}\text { 8. Si me hubieran dado entrenamiento en } \\
\text { el cuidado de la salud bucal, sería capaz de } \\
\text { realizar un mejor cuidado de la salud bucal }\end{array}$ & AE & 81,63 & 18,37 & 0 & 0 & 0 & $1,18(0,39)$ \\
\hline $\begin{array}{l}\text { 9. Si ambos padres tuvieron mala dentadu- } \\
\text { ra, el cepillado y el uso de seda dental no } \\
\text { ayudará }\end{array}$ & LCE & 8,16 & 12,24 & 14,29 & 38,78 & 26,53 & $2,36(1,23)$ \\
\hline $\begin{array}{l}\text { 10. Creo que las prótesis dentales no tienen } \\
\text { que ser removidas }\end{array}$ & LCE & 4,08 & 2,04 & 12,24 & 51,02 & 30,61 & $1,97(0,94)$ \\
\hline $\begin{array}{l}\text { 11. Creo que sé cómo usar la seda dental } \\
\text { correctamente }\end{array}$ & AE & 22,45 & 28,57 & 46,94 & 2,04 & 0 & $2,28(0,84)$ \\
\hline $\begin{array}{l}\text { 12. No se pueden evitar que las enferme- } \\
\text { dades y los medicamentos destruyan los } \\
\text { dientes }\end{array}$ & LCE & 20,41 & 16,33 & 24,49 & 38,78 & 0 & $3,18(1,16)$ \\
\hline $\begin{array}{l}\text { 13. Creo que usar seda dental puede ayudar } \\
\text { a prevenir las enfermedades de las encías }\end{array}$ & $\mathrm{LCl}$ & 34,69 & 36,73 & 20,41 & 8,16 & 0 & $2,02(0,94)$ \\
\hline $\begin{array}{l}\text { 14. Una vez que la enfermedad de las encías } \\
\text { se ha iniciado, es casi imposible detenerla }\end{array}$ & CSO & 2,04 & 20,41 & 26,53 & 36,73 & 14,29 & $2,59(1,03)$ \\
\hline
\end{tabular}




\begin{tabular}{|c|c|c|c|c|c|c|c|}
\hline Preguntas & $\begin{array}{l}\text { Dimen- } \\
\text { sión }\end{array}$ & $\begin{array}{l}\text { Totalmente } \\
\text { de acuerdo } \\
(\%)\end{array}$ & $\begin{array}{l}\text { De } \\
\text { acuerdo } \\
(\%)\end{array}$ & $\begin{array}{l}\text { No } \\
\text { sabe } \\
(\%)\end{array}$ & $\begin{array}{l}\text { Desacuer- } \\
\text { do (\%) }\end{array}$ & $\begin{array}{l}\text { Totalmente } \\
\text { desacuerdo } \\
(\%)\end{array}$ & $\begin{array}{l}\text { Puntaje } \\
\text { promedio } \\
\text { (DS) }\end{array}$ \\
\hline $\begin{array}{l}\text { 15. Creo que sé cómo evitar la candidiasis } \\
\text { oral }\end{array}$ & $\mathrm{AE}$ & 20,41 & 16,33 & 57,14 & 6,12 & 0 & $2,48(0,89)$ \\
\hline $\begin{array}{l}\text { 16. Si las encías sangran cuando usas la seda } \\
\text { dental, por lo general significa que debes } \\
\text { dejar de usarla }\end{array}$ & CSO & 14,29 & 34,69 & 18,37 & 24,49 & 8,16 & $3,22(1,21)$ \\
\hline $\begin{array}{l}\text { 17. Creo que perder dientes es parte del } \\
\text { envejecimiento normal }\end{array}$ & LCE & 28,57 & 40,82 & 10,2 & 12,24 & 8,16 & $3,69(1,24)$ \\
\hline $\begin{array}{l}\text { 18. Incluso si realizas un buen cuidado de } \\
\text { tus dientes, estos se van a caer en la medida } \\
\text { que envejezcas }\end{array}$ & LCE & 22,45 & 28,57 & 6,12 & 38,78 & 4,08 & $3,26(1,30)$ \\
\hline $\begin{array}{l}\text { 19. Creo que nuestros pacientes desean que } \\
\text { les ofrezca ayuda para el cuidado bucal }\end{array}$ & $\mathrm{LCl}$ & 67,35 & 30,61 & 0 & 2,04 & 0 & $1,36(0,60)$ \\
\hline $\begin{array}{l}\text { 20. Creo que visitar al dentista es sólo } \\
\text { necesario cuando se siente dolor }\end{array}$ & CSO & 2,04 & 0 & 2,04 & 36,73 & 59,18 & $1,48(0,73)$ \\
\hline $\begin{array}{l}\text { 21. Si conociera las manifestaciones de las } \\
\text { enfermedades dentales, sería capaz de reali- } \\
\text { zar un mejor cuidado bucal }\end{array}$ & $\mathrm{AE}$ & 69,39 & 28,57 & 2,04 & 0 & 0 & $1,32(0,51)$ \\
\hline $\begin{array}{l}\text { 22. Creo que el cuidado de las prótesis } \\
\text { dentales es menos problemático que cuidar } \\
\text { los dientes naturales }\end{array}$ & CSO & 12,24 & 22,45 & 20,41 & 28,57 & 16,33 & $2,85(1,29)$ \\
\hline $\begin{array}{l}\text { 23. Creo que puedo eliminar en forma satis- } \\
\text { factoria la mayoría de la placa para ayudar } \\
\text { a prevenir las caries y las enfermedades de } \\
\text { las encías }\end{array}$ & $\mathrm{AE}$ & 20,41 & 26,53 & 36,73 & 16,33 & 0 & $2,48(1,0)$ \\
\hline $\begin{array}{l}\text { 24. Creo que un método de cepillado es tan } \\
\text { efectivo como cualquier otro }\end{array}$ & LCE & 26,53 & 22,45 & 22,45 & 22,45 & 6,12 & $3,40(1,27)$ \\
\hline $\begin{array}{l}\text { 25. Creo que las enfermedades de las encías } \\
\text { pueden prevenirse }\end{array}$ & $\mathrm{LCl}$ & 53,06 & 34,69 & 8,16 & 4,08 & 0 & $1,63(0,80)$ \\
\hline $\begin{array}{l}\text { 26. Si las encías sangran cuando cepillas los } \\
\text { dientes, por lo general significa que debes } \\
\text { dejar de cepillarlos }\end{array}$ & CSO & 10,2 & 6,12 & 4,08 & 32,65 & 46,94 & $2(1,3)$ \\
\hline $\begin{array}{l}\text { 27. Creo que nuestros pacientes comen } \\
\text { mejor si ellos tienen una boca sana y limpia }\end{array}$ & $\mathrm{LCl}$ & 75,51 & 16,33 & 6,12 & 2,04 & 0 & $1,34(0,69)$ \\
\hline $\begin{array}{l}\text { 28. Creo que el cepillado de los dientes } \\
\text { puede prevenir las caries }\end{array}$ & $\mathrm{LCl}$ & 69,39 & 24,49 & 2,04 & 2,04 & 2,04 & $1,42(0,81)$ \\
\hline
\end{tabular}

LCI: Locus de control interno; LCE: Locus de control externo; AE: Autoeficacia; CSO: Creencias en Salud Oral 
Tabla 3: Promedios por dimensión del cuestionario, entre cuidadores con y sin capacitación en salud oral

\begin{tabular}{|l|c|c|c|c|c|c|c|c|c|c|}
\hline & \multicolumn{7}{c|}{ Capacitación en salud oral } \\
\hline Global & \multicolumn{7}{c|}{ Si } & & No & \\
\hline & media & rango & DS & media & IC & DS & media & IC & DS & p \\
\hline Locus control interno & 11,10 & $7-18$ & 2,89 & 9,83 & $8,38-11,28$ & 2,91 & 11,83 & $10,86-12,81$ & 2,65 & 0,0178 \\
\hline Locus control externo & 20,6 & $14-32$ & 3,66 & 20,83 & $18,66-22,99$ & 4,35 & 20,45 & $19,25-21,64$ & 3,26 & 0,729 \\
\hline Autoeficacia & 14,6 & $9-20$ & 2,68 & 13,55 & $12,38-14,72$ & 2,35 & 15,16 & $14,16-16,15$ & 2,72 & 0,0423 \\
\hline Creencias en Salud Oral & 18,9 & $8-33$ & 4,29 & 18,38 & $16,35-20,42$ & 4,08 & 19,22 & $17,59-20,85$ & 4,44 & 0,5166 \\
\hline
\end{tabular}

\section{Discusión}

El objetivo de este estudio fue analizar las creencias relacionadas al cuidado de la salud oral en cuidadores de adultos mayores institucionalizados de la ciudad de Antofagasta, Chile en 2019. Como fortalezas de esta investigación está la muestra obtenida, ya que la totalidad de los cuidadores de los ELEAM de Antofagasta aceptaron participar y solo se excluyó a uno, porque llevaba ejerciendo menos de dos meses, por lo cual los resultados son representativos de la realidad estudiada.

Respecto a las características de estos cuidadores, todas correspondieron a mujeres. Esto se correlaciona con la literatura, la cual describe que el rol de cuidado está asociado a la mujer, lo cual se explica por patrones culturales que llevan a la feminización del cuidado y que también fue observado en los estudios de Godoy J et al. ${ }^{(11)}$, Garrido-Urrutia C et al. ${ }^{(4,14)}$, FuentesP C et al. ${ }^{(10)}$ y Cornejo-Ovalle $\mathrm{M}$ et al. ${ }^{(5)}$, en cuidadores de personas mayores. Respecto a la edad de los cuidadores, el promedio fue de 39 años, resultado similar al encontrado en Chile por Godoy J et al. ${ }^{(11)}$ y Garrido-Urrutia C et al. ${ }^{(14)}$ en cuidadores de adultos mayores institucionalizados en de los ELEAM de Valparaíso y de una residencia en la ciudad de Santiago, respectivamente, pero inferior al de Fuentes-P $\mathrm{C}$ et al. ${ }^{(10)}$ quienes evaluaron cuidadores de pacientes discapacitados y adultos mayores institucionalizados de la región del Bío-Bío, encontrando una edad promedio de 52,7 años en los cuidadores. Estas diferencias se pueden expli- car porque la mayoría de los cuidadores en el estudio de Fuentes-P C et al. ${ }^{(10)}$, corresponden a cuidadores informales, siendo los esposos/as en mayor porcentaje que se encargaban del cuidado. En relación con el nivel educacional de los cuidadores de este estudio, cerca del 86\% tiene un nivel educacional de enseñanza media o superior y se destaca que casi el 33\% de ellos ha tenido estudios superiores ya sea completos o incompletos, lo cual también puede relacionarse a que la muestra en nuestro estudio estuvo conformada en su totalidad por cuidadores formales, que por definición debiesen tener capacitación para cumplir dicho rol, por el cual perciben una remuneración.

Respecto al tiempo como cuidador, el 46,94\% ha ejercido como cuidador entre uno y dos años y $36,73 \%$ tres o más años, sin embargo, solo el $36,73 \%$ relata haber recibido capacitación en salud oral, este resultado es algo superior al reportado por Godoy J et al. ${ }^{(11)}$ y Fuentes-P C et at. ${ }^{(10)}$, en dónde respectivamente, solo el $24 \%$ y $25 \%$ de los cuidadores formales relataron tener formación en cuidado bucal. Sin embargo, esto continúa siendo una baja capacitación en el área salud bucal. De hecho, al analizar la autopercepción de necesidad de capacitación para realizar el cuidado bucal de un adulto mayor, se evidenció que prácticamente la totalidad de los cuidadores en este estudio percibe la necesidad de capacitarse en esta área. Esto es relevante, porque sería un indicador positivo de que una intervención educativa en salud bucal en este grupo sería bien aceptada. 
En cuanto al análisis de las creencias relacionadas al cuidado de la salud oral, se observó porcentaje altos de respuestas "no sabe" en preguntas de la dimensión autoeficacia, que dicen relación a la creencia de saber cómo tratar las enfermedades de la mucosa oral $(71,43 \%)$, cómo utilizar de la seda dental correctamente $(46,94 \%)$, y cómo evitar la candidiasis oral $(57,14 \%)$ y en la creencia de ser capaz de poder eliminar correctamente la placa bacteriana para prevenir las caries y enfermedades de las encías (36,73\%). La dimensión autoeficacia es importante pues permite identificar circunstancias a las que los cuidadores pueden sentirse incapaces de afrontar o lidiar ${ }^{(15)}$.

Por otro lado, se encontraron creencias desfavorables a la salud bucal en la dimensión creencias en salud oral y locus de control externo. En la dimensión creencias en salud oral, la mayoría cree que debieran ser los mismos pacientes los que avisen cuando necesiten ayuda en su cuidado de salud oral $(55,11 \%)$, creen que el flúor solo tiene efectos beneficiosos para la población pediátrica (el 40,82\% tiene creencias desfavorables y un $36,73 \%$ no sabe) y además creen que si las encías sangran cuando utilizan seda dental, por lo general significa que deben dejar de usarla $(48,98 \%)$.

En la dimensión locus de control externo, la mayoría de ellos creen que no se puede evitar que las enfermedades y los medicamentos destruyan los dientes (un 36,74\% tiene creencias desfavorables y un $24,49 \%$ no sabe), que perder dientes es parte del envejecimiento normal $(69,39 \%)$, que si realizan un buen cuidado de los dientes, estos se caerán igual a medida que se envejezca $(51,02 \%)$ y que un método de cepillado es tan efectivo como cualquier otro (un 48,98\% tiene creencias desfavorables y un $22,45 \%$ no sabe). El locus de control externo se da cuando el individuo percibe o cree que otros controlan sus decisiones en salud y su estado de salud. Se ha visto que el locus de control puede predecir conductas en salud y que intervenciones de educación para la salud son más exitosas cuando los sujetos que participan tienen locus de control interno ${ }^{(13)}$.

Finalmente, se evidenció que aquellos cuidadores que si han recibido capacitación tienen significativamente promedios inferiores, es decir, favorables a la salud bucal, en las dimensiones locus control interno y autoeficacia $(\mathrm{p}<0,05)$. Lo cual podría ser indicador de que una intervención educativa en salud oral que se enfoque en las creencias desfavorables o erróneas descritas en este grupo de cuidadores podría mejorar y ser favorables a la salud bucal de los adultos mayores bajo su cuidado. De hecho, una revisión sistemática de la literatura respecto al efecto de los programas de educación en salud bucal en cuidadores de personas mayores (1), evidenció que estos mostraron mejorías en parámetros bucales en las personas mayores institucionalizadas y en sus niveles de higiene bucal. Además, demostraron que el educar respecto a la salud bucal y su asociación a otras patologías puede mejorar la actitud de los cuidadores hacia la importancia de la salud bucal de las personas institucionalizadas. Al mismo tiempo, intervenciones que consistían en varias sesiones de educación para la salud mostraron ser mucho más efectivas en aumentar el conocimiento y los procedimientos prácticos de higiene bucal.

A partir de esto podemos determinar que la intervención educativa a realizar en este grupo estudiado tiene que enfocarse en los aspectos analizados a través de sus creencias y debiera tener un componente teórico y práctico-participativo buscando aumentar la autoeficacia de los cuidadores, pues mayores niveles de autoeficacia se asocian a mayor probabilidad de reproducir comportamientos favorables a la salud ${ }^{(15)}$. Respecto a los resultados o creencias desfavorables, mayoritariamente los encontramos en las dimensiones de locus de control externo y creencias en salud oral. De acuerdo con esto, es importante considerar en el diseño de la intervención educativa en salud desarrollar técnicas que influyan en sujetos con una orientación de locus de control externo para que se vuel- 
van más orientados hacia un locus interno de control, pero además considerar que para que ocurran cambios que favorezcan a la salud, la educación para la salud debe actuar como el incentivo para el cambio ${ }^{(13)}$.

En este sentido, el análisis mostrado en este estudio es relevante, ya que este grupo en la ciudad de Antofagasta en el norte de Chile hasta el momento no había sido evaluado respecto a sus creencias relacionadas a la salud oral, por lo cual los resultados de nuestra investigación son un aporte a la evidencia para poder comprender las creencias de los cuidadores de adultos mayores en los ELEAM de Antofagasta, en miras de establecer intervenciones educativas que sean de apoyo al rol que cumplen.

\section{Conclusiones}

Los cuidadores de adultos mayores institucionalizados de la ciudad de Antofagasta en el norte de Chile tienen baja capacitación para el cuidado de la salud bucal, existiendo creencias desfavorables al cuidado de la salud bucal de las personas mayores institucionalizados. Es necesario diseñar intervenciones de educación para la salud bucal en este grupo que permitan mejorar las creencias relacionadas al cuidado de la salud oral.

\section{Agradecimientos}

Los autores desean agradecer a la Dra. Casandra Bracamonte Wolf, directora de la carrera de Odontología de la Universidad Pedro de Valdivia sede Antofagasta en el año 2019, por gestiones realizadas con los ELEAM de Antofagasta, y a cada uno de los directores técnicos de los ELEAM por aceptar participar en este estudio.

\section{Referencias}

1. Wang TF, Huang CM, Chou C, Yu S. Effect of Oral Health Education Programs for Caregivers on Oral Hygiene of the Elderly: A Systemic Review and Meta-Analysis. Int J Nurs Stud. 2015; 52(6):1090-6.

2. Weening-Verbree L, Huisman-de Waal G, van Dusseldorp L, van Achterberg T, Schoonhoven L. Oral Health Care in Older People in Long Term Care Facilities: A Systematic Review of Implementation Strategies. Int J Nurs Stud. 2013; 50(4):569-82.

3. Baumgartner W, Schimmel M, Müller F. Oral health and dental care of elderly adults dependent on care. Swiss Dent J. 2015;125(4):417-26.

4. Garrido-Urrutia C, Romo-Ormazábal F, Araya D, Muñoz-López D, Espinoza I. Cuidado bucal en mayores dependientes de un programa de cuidados domiciliarios. Rev. Clin. Periodoncia Implantol. Rehabil. Oral. 2016; 9(2):140-145.

5. Cornejo-Ovalle M, Costa-de-Lima K, Péres G, Borrell C, Casals-Peidro E. Oral Health Care Activities Performed by Caregivers for Institutionalized Elderly in Barcelona-Spain. Med Oral Patol Oral Cir Bucal. 2013;18(4):e641-9.

6. Riquelme-Pérez M. Metodología de educación para la salud. Rev Pediatr Aten Primaria. 2012; (21):7782.

7. Muñoz AD, Flores P, Cueto A, Barraza A. Salud bucodental de postrados severos. Int J Odontostomat. 2012;6:181-7.

8. Quinteros ME, Nunez - Franz L. Salud oral en adultos mayores postrados. Int J Odontostomat. 2014;8:337-43.

9. Araya C, Oliva P, Canete E, Zapata H. Salud oral en pacientes adultos mayores del programa postrados. CESFAM Los Volcanes, Chillán. Ciencia Ahora. 2009;23:70-9. 
10. Fuentes-P C, Escobar-I C, Muñoz-M P, Ottone NE. Creencias en Salud Oral de Cuidadores de Pacientes Discapacitados y Adultos Mayores Institucionalizados de la Comuna de Quilaco, Región del BioBío. Int. J. Odontostomat. 2015; 9 ( 2 ): 301-306.

11. Godoy J, Rey P. Conocimiento en higiene oral de cuidadores establecimientos geriátricos de larga estadía, Valparaíso. Rev. Clin. Periodoncia Implantol. Rehabil. Oral. 2019;12(1):12-14.

12. Garrido-Urrutia C, Espinoza-Santander I, Romo-Ormazabal F. Traducción al español y validación del cuestionario de creencias en salud bucal para cuidadores (DCBS-SP). Rev Esp Salud Publica. 2010; 84(4):409-16.

13. Misrachi C, Sassenfield A, Abarca G, Rojas G. Fundamentos psicosociales de las conductas en salud oral. Rev Dent Chile. 2006; 07(3): 30-34.

14. Garrido-Urrutia C, Romo-Ormazabal F. Espinoza-Santander I \& Medics-Salvo D. Oral Health Practices and Beliefs Among Caregivers of the Dependent Elderly. Gerodontology,. 2012; 29(2):e742-7.

15. Kakudate N, Morita M, Fukuhara S, Sugai M, Nagayama M, Kawanami M \& Chiba I. Application of self-efficacy theory in dental clinical practice. Oral Dis. 2010; 16(8):747-52.

\section{Nota conflictos de interés:}

Los autores declaran no tener conflictos de interés respecto a la autoría y/o publicación del artículo.

\section{Nota contribución de los autores:}

1. Concepción y diseño del estudio

2. Adquisición de datos

3. Análisis de datos

4. Discusión de los resultados

5. Redacción del manuscrito

6. Aprobación de la versión final del manuscrito

JG ha contribuido en: 1, 2, 4, 5 y 6 .

ER ha contribuido en: 1,4 y 6 .

CG-U: $1,3,4,5$ y 6 .

\section{Nota fuente de financiamiento:}

Autofinanciado

\section{Nota aprobación Comité de Ética:}

Estudio aprobado por el Comité de Ética de la Universidad Pedro de Valdivia

\section{Nota de aceptación:}

Este artículo fue aprobado por la editora de la revista Mag. Dra. Vanesa Pereira-Prado. 